\title{
Suturing versus flowering technique of Bruhat after fimbrioplasty for endometriosis-related infertility
}

\author{
Mohamed F. Mitwally • Anusha Thotakura • \\ Omar Abuzeid • Mohammad Ashraf • \\ Michael P. Diamond • Mostafa Abuzeid
}

Received: 15 July 2008 / Accepted: 25 August 2008 / Published online: 2 October 2008

(C) Springer-Verlag 2008

\begin{abstract}
The objective of this study is to compare the effectiveness of two surgical techniques (suturing versus flowering of Bruhat) after fimbrioplasty for treatment of distal tubal pathology in infertile women with endometriosis. This is a historical cohort study with 12 months of follow-up comparing pregnancy rates achieved spontaneously or after controlled ovarian hyperstimulation (COS) with intrauterine insemination (IUI) in infertile women with endometriosis who underwent laparoscopic fimbrioplasty. A total of 154 patients with endometriosis-related infertility (pelvic inflammatory disease was excluded by absent history and negative Chlamydia trachomatis serology) had fimbrioplasty using salpingostomy procedure for treatment of distal tubal pathology. The edges of the fimbrial ostium were everted
\end{abstract}

M. Ashraf $\cdot$ M. Abuzeid

Division of Reproductive Endocrinology and Infertility,

Department of Obstetrics and Gynecology,

Hurley Medical Center,

Flint, MI, USA

M. Ashraf

e-mail: mohdashrafmd@aol.com

\author{
A. Thotakura \\ Department of Obstetrics and Gynecology, \\ Hurley Medical Center, \\ Flint, MI, USA \\ e-mail: anushavalluru@yahoo.com \\ M. Ashraf $\cdot$ M. Abuzeid \\ College of Human Medicine, Michigan State University, \\ East Lansing, MI, USA
M. F. Mitwally • O. Abuzeid · M. Ashraf • M. Abuzeid
IVF-Michigan,
Rochester Hills, MI, USA
M. F. Mitwally
e-mail: mmitwally@yahoo.com

using either the flowering technique of Bruhat or 6-0 Vicryl sutures (intracorporeal knot) using microsurgical techniques. Forty-six patients had flowering (group 1) and 108 had suturing (group 2) technique, followed by timed intercourse and/or ovarian hyperstimulation with insemination (COSIUI) with follow-up until pregnancy or at least 12 months. There was no significant difference in patients' characteristics (age, infertility duration, and endometriosis stage) between the two groups. The pregnancy rate per cycle or per patient and cumulative pregnancy rates were not significantly different between the two groups. There appears to be no advantage of the suturing technique over flowering after salpingostomy for fimbrioplasty. The latter method is easier to learn and requires less operative time to perform.

\section{O. Abuzeid \\ e-mail: AR9935@wayne.edu}

M. F. Mitwally $\cdot$ M. P. Diamond

Division of Reproductive Endocrinology and Infertility,

Department of Obstetrics and Gynecology,

Wayne State University,

Detroit, MI, USA

M. P. Diamond

e-mail: mdiamond@med.wayne.edu

M. Abuzeid ( $\square)$

Hurley Medical Center,

Two Hurley Plaza, Suite 209,

Flint, MI 48504, USA

e-mail: reprod1@hurleymc.com

M. F. Mitwally

Division of Reproductive Endocrinology and Infertility,

Department of Obstetrics,

Gynecology \& Women's Health,

University of Minnesota,

Minneapolis, MN, USA 
Keywords Endometriosis · Fimbrioplasty . Flowering technique of Bruhat · Infertility · Intrauterine insemination - Laparoscopic conservative surgery

\section{Introduction}

Patent fallopian tubes are a prerequisite for spontaneous human fertility. However, patency alone is not enoughnormal function is crucial. Although patients often view them as either open or "blocked," the fallopian tubes are highly specialized organs. They have a critical role in oocyte pick up, as well as transportation of oocytes, sperm, and the embryo [1]. Fallopian tube obstruction occurs in $12 \%$ to $33 \%$ of infertile couples. There are several causes of tubal damage including pelvic infection, endometriosis, and adhesions following surgery [1].

There are different diagnostic modalities for detection of tubal damage including hysterosalpingography and ultrasonography to detect dilated tubes when filled with fluid (hydrosalpinx), as well as laparoscopy. Direct visualization during laparoscopy is the gold standard for the diagnosis of the type and extent of damage to the serosal and distal aspects of the tubes.

Complete tubal occlusion is rarely caused by pelvic endometriosis. However, fimbrial stenosis and tubal distortion with limitation of fimbrial mobility are more frequent pathologies associated with endometriosis. Surgical treatment by adhesiolysis in addition to correction of fimbrial pathology potentially results in promising outcome; i.e., high rates of spontaneous pregnancies. This is particularly true in patients with endometriosis due to the absence of tubal endothelial damage. However, this is not usually the case in patients with history of pelvic infection, e.g., pelvic inflammatory disease (PID), in which tubal endothelial damage is often encountered [1,2].

Distal tubal pathology (as classified by Gomel) includes (1) perifimbrial adhesions, with or without associated intrainfundibular adhesions; (2) partial or complete agglutination of the fringes (phimosis); (3) serosal covering of the fringes leaving only a small tubal opening in which, in rare instances, the covering is total, leading to a complete occlusion (hard to differentiate from a true hydrosalpinx); (4) prefimbrial phimosis [3].

Such fimbrial pathology is usually amenable to surgical correction with a specific procedure, frequently called "fimbrioplasty." However, techniques of fimbrioplasty can be classified as: (1) adhesiolysis for fimbrial adhesions only; (2) deglutination of the fringes; and (3) salpingostomy, when a serosal incision is required $[4,5]$.

To minimize reobstruction of the tubal ostium after salpingostomy, everting the edges of the fimbrial ostium is often advocated. This can be done by suturing the edges of the ostium to the surrounding wall of the tube (suturing technique) or by everting the edges of the fimbrial ostium by heating the serosal surface of the tube surrounding the ostium using electrical or laser energy (flowering technique). The suturing technique is the gold standard during laparotomy and microtubal surgery [3], while the flowering technique is utilized during operative laparoscopy as being an easier technique to learn.

In this historical cohort study, we evaluated the reproductive outcomes (achievement of pregnancy, rates of live birth, miscarriage, and ectopic pregnancy), in infertile women with endometriosis (without evidence of PID) who underwent laparoscopic fimbrioplasty for surgical management of fimbrial pathology. The primary aim of the study was to compare such reproductive outcomes between two surgical techniques (suturing versus flowering of Bruhat) done for everting the edges of the fimbrial ostium after fimbrioplasty.

\section{Materials and methods}

This is a historical cohort study conducted after obtaining approval by the local institutional research board of Wayne State University. The study was conducted at an infertility center (IVF-Michigan). After excluding patients with PID, hydrosalpinges, or absent tube on one side, the study included a group of patients with endometriosis, selected out of a cohort of 650 infertile women who underwent conservative laparoscopic surgery for tubal factor infertility in the period from 1992 to 1999 . PID was excluded by a negative history and negative Chlamydia trachomatis serology. Patients with peritubal adhesions and/or fimbrial agglutination but no distal tubal pathology requiring salpingostomy were not included in this study. Patients with significant male factor infertility were excluded. The selected cohort of patients included 154 infertile women (66 with stage I, 41 with stage II, 33 with stage III, and 14 with stage IV endometriosis) who had distal tubal disease (fimbrial pathology). In all patients, both tubes were patent (patients who had neosalpingostomy for blocked tubes were not included).

Conservative laparoscopic surgery was done by the same reproductive surgeon (M.I.A.) under similar operative sittings in all patients included staging (American Society for Reproductive Medicine classification) [6], fulguration of endometriosis, lysis of adhesions when present around the tubes (salpingolysis) and around the ovaries (ovariolysis), and excision of endometrioma when present in patients with stage III or stage IV endometriosis.

All patients had distal tubal pathology in the form of fimbrial agglutination and fimbrial ostium blunting and/or phimosis and/or prefimbrial phimosis requiring fimbrioplasty with salpingostomy. Fimbrioplasty and salpingostomy procedures were done by incising the antimesenteric 
side (1- to $1.5-\mathrm{cm}$ length) of the stenosed fimbrial ostium, prefimbrial phimosis, and fimbrial blunting using monopolar needle tip cautery (Elmed, Chicago, IL, USA) while probing the fimbrial end by a Teflon probe (Elmed, Chicago, IL, USA; Fig. 1a,b). If fimbrial agglutination was found, deglutination of fimbrial fringes was performed. The same surgical techniques and procedures were applied in all patients.

Following fimbrioplasty by salpingostomy, in order to keep the fimbrial ostium adequately open and prevent tubal occlusion, a procedure was done to keep the edges of the fimbrial ostium everted. This was done either by suturing the edges of the fimbrial ostium to the surrounding tubal serosa using 6-0 Vicryl sutures (intracorporeal knot) using microsurgical principles and instruments with 3-mm needle holders (Koh Micro Instruments, Karl Storz Endoscopy, Culver City, CA, USA; Fig. 2) or by the technique of heating the serosal surfaces of the tube around the fimbrial
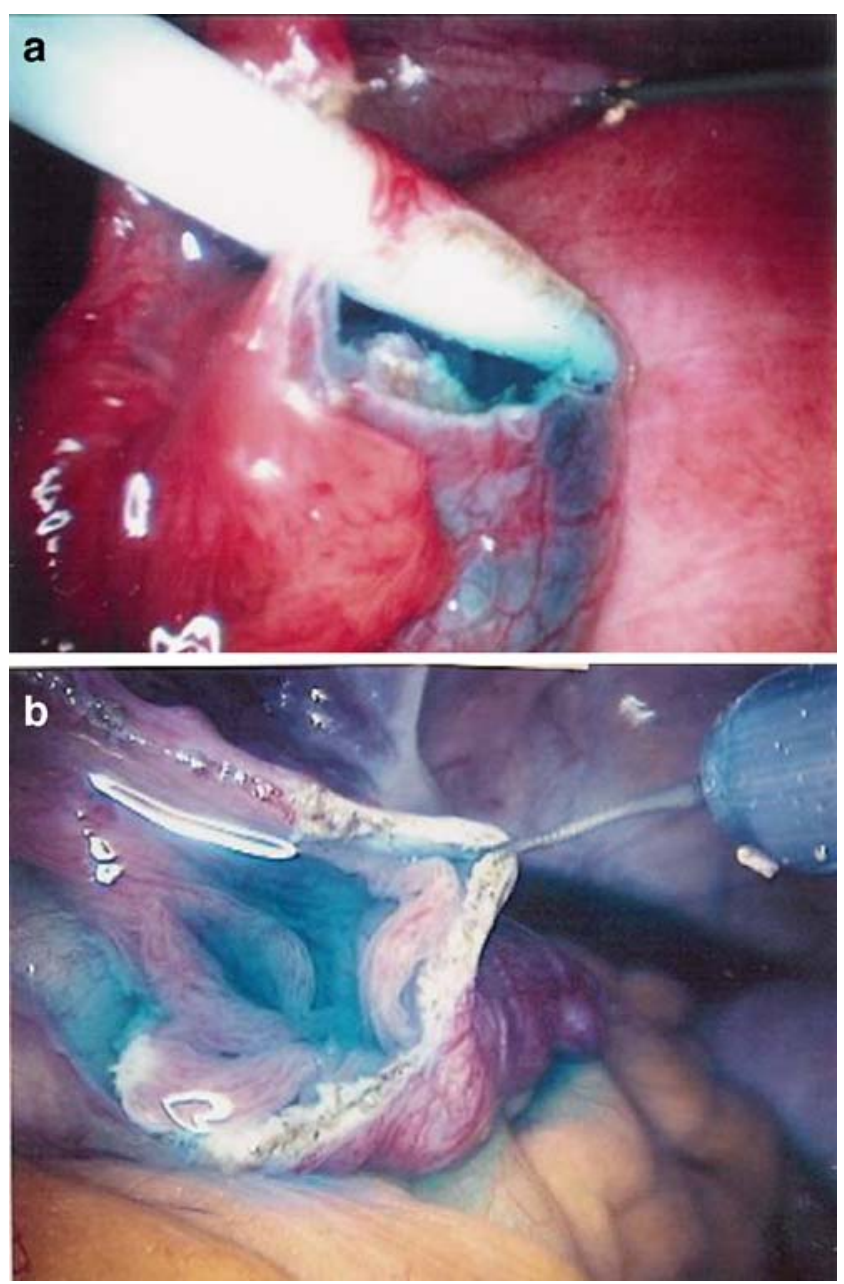

Fig. 1 a The technique of salpingostomy for fimbrioplasty using Teflon Probe and monopolar needle tip. b The new ostium after salpingostomy for fimbrioplasty

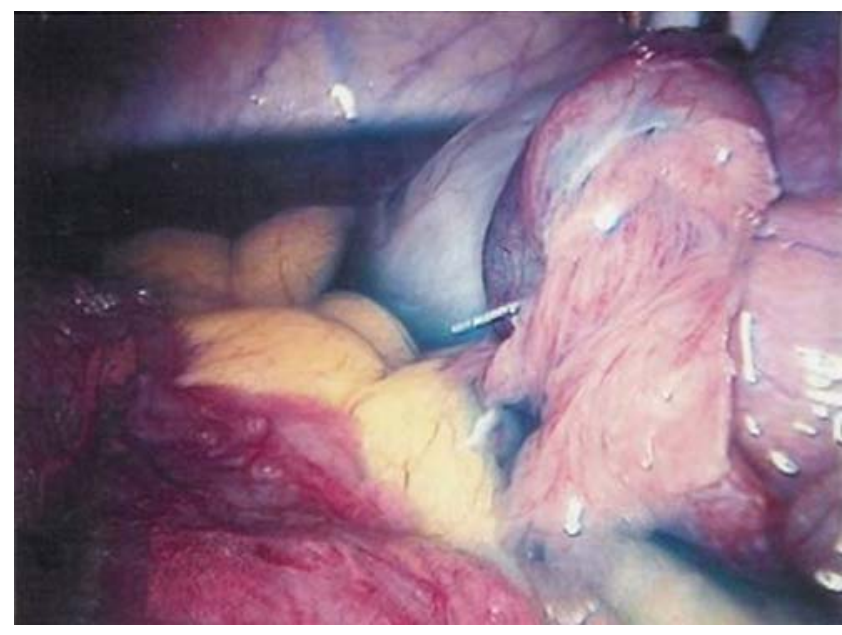

Fig. 2 The edges of the new ostium everted using two 6-0 Vicryl sutures

ostium (flowering of Bruhat) [7] using Argon Beam coagulator (Birtcher Medical System, Irvine, CA, USA; Fig. 3). All through the procedure, irrigation and suction were performed using heparinized Lactated Ringer's solution $(5,000 \mathrm{IU}$ in 11$)$.

These cases were done between 1992 and 1999. During the first 4 years of that period (between 1992 and 1995), all of the cases were done according to the suturing technique, while, along the last 3 years (between 1997 and 1999), all the cases were done by the flowering technique of Bruhat with a transitional period of 1 year (1996), during which a shift from the suturing to flowering technique occurred. The decision was empirically taken based on noticing advantages of the flowering technique of Bruhat as regards the decreased operative time without deleterious effect on the outcome (achievement of pregnancy) following surgery.

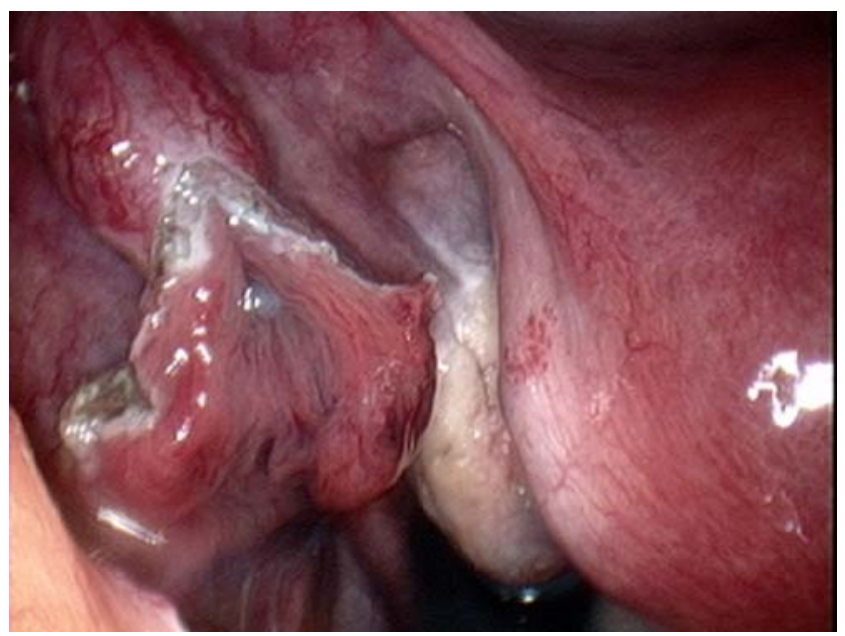

Fig. 3 The edges of the new ostium everted using the flowering technique of Bruhat 
All patients had either technique of suturing or flowering of Bruhat on one (unilateral) or both tubes (bilateral). None of the patients had both techniques combined at the same time. Patients who had unilateral fimbrioplasty had a tube on the other side that was free from fimbrial pathology.

Patients were subgrouped according to the side of fimbrioplasty into unilateral and bilateral and the outcomes were compared between the two techniques of suturing and flowering separately.

After surgery, patients were offered the option of trying on their own to achieve spontaneous conception or to start infertility intervention in the form of controlled ovarian stimulation (COS) with gonadotropin injections in conjunction with intrauterine insemination (IUI). In patients with associated ovulatory disorder, clomiphene citrate was used for ovulation induction in the first instance. One hundred seven patients tried to conceive spontaneously (Table 1) while 65 patients tried COS with IUI (Table 2). Forty-seven patients tried COS with IUI from the outset, while 18 patients first tried spontaneous conception then decided to proceed with COS with IUI. COS was performed using midluteal gonadotropin-releasing hormone agonist for pituitary downregulation followed by gonadotropin injections at a dose from 75 to $300 \mathrm{IU} /$ day (depending on the clinical profile of the patient including her age, body mass index, and day 3 follicle-stimulating hormone level, as well as response in a prior stimulation cycle). Gonadotropin injections started on day 3 of the menstrual cycle followed by human chorionic gonadotrophin (10,000 IU) administration when the dominant follicle reached a mean diameter of $17 \mathrm{~mm}$, followed by insemination $42 \mathrm{~h}$ later.

Patients were followed up until pregnancy was achieved or a maximum of 12 months elapsed. Achieved pregnancies were followed until delivery or pregnancy loss (miscarriage or ectopic pregnancy). The choice as regards the duration of trying to achieve spontaneous pregnancy or to undergo ovarian stimulation with IUI was chosen by the couples empirically after providing them with the success chances and considering other factors including the woman's age and insurance coverage, as well as duration of infertility.
The rates of spontaneous pregnancy per cycle and pregnancy rate per treatment cycle by gonadotropin ovarian stimulation with IUI were calculated and compared between the two study groups (suturing and flowering techniques) both in unilateral and bilateral fimbrioplasty subgroups. The outcomes of achieved pregnancies were also compared similarly.

\section{Statistical analysis}

Statistical analyses were performed using Student's $t$ test and chi square test (continuous and dichotomous variables, respectively) considering $P$ value $<0.05$ as statistically significant where appropriate. The statistical tests were performed with SigmaStat for Windows version 1.0 software (Sigma Stat Software High Edit Professional Copyright ${ }^{\circledR}$ 1993, Micro Help Inc. and Heiler Software GmbH, San Rafael, CA, USA). Kaplan-Meier analysis was used to calculate cumulative conception rate (CCR) using SPSS for Windows version 11.5 software (SPSS Inc., Copyright $(C$ 2002, 511210 Software Publishers, Chicago, IL, USA).

\section{Results}

Group mean age, mean duration of infertility, and endometriosis stage were not different between the two study groups (suturing versus flowering) or between the two corresponding subgroups (unilateral and bilateral fimbrioplasty), as shown in Table 1.

The outcomes of cycles of trying spontaneous conception and COS with IUI are displayed in Tables 2 and 3, respectively. There was no statistically significant difference between the two fimbrioplasty techniques, as regards pregnancy, delivery, and miscarriage rates. None of the patients who had flowering fimbrioplasty experienced ectopic pregnancies, while two patients in the suturing technique had ectopic pregnancies. It is important to remember that patients tried spontaneous conception and/or ovarian stimulation with IUI for different number of cycles. The absence of significant

Table 1 Characteristic of patients who underwent different methods of fimbrioplasty

\begin{tabular}{|c|c|c|c|c|}
\hline & \multicolumn{2}{|c|}{ Unilateral fimbrioplasty } & \multicolumn{2}{|l|}{ Bilateral fimbrioplasty } \\
\hline & Flowering technique & Suturing technique & Flowering technique & Suturing technique \\
\hline Number of patients & 30 & 50 & 16 & 58 \\
\hline Age (years) & $30.5 \pm 4.8$ & $31.4 \pm 4.1$ & $31.8 \pm 3.8$ & $30.6 \pm 4.1$ \\
\hline Duration of infertility (years) & $3.8 \pm 3.1$ & $4.1 \pm 2.9$ & $3.4 \pm 3.5$ & $3.8 \pm 2.4$ \\
\hline \# patients with stage I endometriosis (\%) & $14(46)$ & $21(42)$ & $6(38)$ & $25(43)$ \\
\hline \# patients with stage II endometriosis (\%) & $6(20)$ & $10(20)$ & $5(31)$ & $20(34)$ \\
\hline \# patients with stage III endometriosis (\%) & $5(17)$ & $13(26)$ & $4(25)$ & $11(19)$ \\
\hline \# patients with stage IV endometriosis (\%) & $5(17)$ & $6(12)$ & $1(6)$ & $2(4)$ \\
\hline
\end{tabular}


Table 2 Pregnancy outcome of spontaneous cycles

\begin{tabular}{|c|c|c|c|c|}
\hline & \multicolumn{2}{|c|}{ Unilateral fimbrioplasty } & \multicolumn{2}{|c|}{ Bilateral fimbrioplasty } \\
\hline & $\begin{array}{l}\text { Flowering } \\
\text { technique }\end{array}$ & $\begin{array}{l}\text { Suturing } \\
\text { technique }\end{array}$ & $\begin{array}{l}\text { Flowering } \\
\text { technique }\end{array}$ & $\begin{array}{l}\text { Suturing } \\
\text { technique }\end{array}$ \\
\hline Number of patients & 12 & 37 & 11 & 47 \\
\hline Number of cycles & 139 & 372 & 95 & 412 \\
\hline Number of achieved pregnancies & 4 & 9 & 4 & 12 \\
\hline Pregnancy rate per cycle, $\%$ & 2.9 & 2.4 & 4.2 & 2.9 \\
\hline Pregnancy rate per patient, $\%$ & 33 & 24 & 36 & 26 \\
\hline Number of live births & 3 & 8 & 3 & 11 \\
\hline Delivery rate per cycle, $\%$ & 2.2 & 2.2 & 3.2 & 2.7 \\
\hline Delivery rate per patient, $\%$ & 25 & 22 & 27 & 23 \\
\hline Number of miscarriages (\%) & $1(25.1)$ & $1(11.1)$ & $1(25.1)$ & $1(8.3)$ \\
\hline Number of ectopic pregnancies (\%) & 0 & 0 & 0 & 0 \\
\hline $\mathrm{CCR}, \%$ & 40 & 23 & 45 & 33 \\
\hline
\end{tabular}

differences between the two techniques was maintained after controlling for the age, duration of infertility, and the stage of endometriosis (data not shown). CCR after 12 months in spontaneous cycles and COS with IUI after flowering or suturing techniques in both unilateral and bilateral subgroups are illustrated in Tables 2 and 3, respectively. There was no significant difference in CCR between the flowering and suturing techniques in both unilateral and bilateral subgroups after spontaneous conception or COS with IUI. When CCR was calculated for all methods of conception (spontaneous and IUI + COS), it was $71 \%$ and $40 \%$ for the flowering and suturing techniques, respectively, in the unilateral subgroups and $58 \%$ and $49 \%$ for flowering and suturing techniques, respectively, in the bilateral subgroups.

\section{Discussion}

This study did not find significant differences in the reproductive outcomes following laparoscopic conservative surgery for infertile women with endometriosis, when two different fimbrioplasty techniques (suturing versus flowering) were applied for management of associated distal tubal pathology. After controlling for the side of fimbrial pathology (unilateral or bilateral), woman's age, and duration of infertility, as well as stage of endometriosis, there was no significant difference in the birth rate per cycle or per patient (spontaneous pregnancy or pregnancies achieved after COS with IUI). In addition, there was no significant difference in CCR between the two techniques in each group (flowering versus suturing) or subgroups (unilateral versus bilateral). There were only two cases of ectopic pregnancies in the group of suturing fimbrioplasty (one in the unilateral and one in the bilateral fimbrioplasty subgroups). The small number does not allow drawing strong conclusions regarding the risk of ectopic pregnancy following suturing fimbrioplasty. However, the overall low rate of ectopic pregnancy among all achieved pregnancies (two cases out of 60 pregnancies or $3.3 \%$ ) supports the notion that fimbrioplasty, in women with endometriosis without a history of PID, does not seem to increase the risk of ectopic pregnancy to significantly high levels.

Table 3 Outcome of cycles of controlled ovarian stimulation with intrauterine insemination

\begin{tabular}{|c|c|c|c|c|}
\hline & \multicolumn{2}{|c|}{ Unilateral fimbrioplasty } & \multicolumn{2}{|l|}{ Bilateral fimbrioplasty } \\
\hline & Flowering technique & Suturing technique & Flowering technique & Suturing technique \\
\hline Number of patients & 10 & 19 & 6 & 30 \\
\hline Number of cycles & 13 & 28 & 11 & 40 \\
\hline Number of achieved pregnancies & 6 & 8 & 4 & 13 \\
\hline Pregnancy rate per cycle, $\%$ & 46.2 & 28.6 & 36.4 & 32.5 \\
\hline Pregnancy rate per patient, $\%$ & 60 & 42 & 67 & 33 \\
\hline Number of live births & 3 & 6 & 3 & 8 \\
\hline Delivery rate per cycle, $\%$ & 23.1 & 21.4 & 27.3 & 20.1 \\
\hline Delivery rate per patient, $\%$ & 30 & 32 & 50 & 20 \\
\hline Number of miscarriages (\%) & $3(50.1)$ & $1(12.5)$ & $1(16.7)$ & $4(50.1)$ \\
\hline Number of ectopic pregnancies (\%) & 0 & $1(12.5)$ & 0 & $1(12.5)$ \\
\hline $\mathrm{CCR}, \%$ & 67 & 61 & 56 & 71 \\
\hline
\end{tabular}


There are several valuable studies that reported encouraging pregnancy rates following fimbrioplasty in women with tubal infertility, particularly those with early stages of tubal infertility [8-10]. However, these studies suffered from the significant problem of heterogeneity of the infertile population included, in particular, including women with tubal pathology secondary to pelvic infection and endometriosis. In addition, the surgical conditions including different surgeons and various techniques make results drawn from these studies difficult to interpret.

In our study, we compared the reproductive outcome after fimbrioplasty in a selected population of infertile women with endometriosis-related fimbrial pathology using two different techniques for everting the edges of the new ostium (suturing versus flowering).

In this study, we elected to include only patients with endometriosis and fimbrial pathology, as data are lacking on reproductive outcome after laparoscopic fimbrioplasty in such a group of patients. Recent data from our unit suggest the presence of fimbrial pathology in many patients with early stages of endometriosis [11]. Whenever fimbrial pathology was detected, it was corrected surgically at the same time of diagnostic laparoscopy. Whenever salpingostomy was performed in cases of fimbrial blunting, fimbrial phimosis, or prefimbrial phimosis (as in all patients in our study), eversion of the fimbrial ostium was performed to avoid partial reocclusion. It was observed that the edges of the new ostium were always seen almost contacting each other (Fig. 1a), which may increase the chances of reattachment to each other. Such a principal of eversion after salpingostomy was always advocated during laparotomy and microtubal surgery using 6-0 Vicryl [3]. When we started performing operative laparoscopy, we decided to advocate the same technique. Since 1996, we started using the flowering technique in some patients, and since 1997 the technique of suturing was abandoned in favor of flowering technique for its simplicity and the fact that initial results suggested no compromise in pregnancy rates.

Our study has some limitation with respect to the design being retrospective and nonrandomized. In addition, any effect across time, e.g., experience, may be confounded with treatment effect. The effect of the latter issue is limited, as all the patients were operated on by the same surgeon under the same operative setting. In addition, the comparison is between two techniques; the first to be used (suturing) requires experience and expertise to start with. Switching to the flowering technique was a switch to a much simpler and easier technique to learn. Furthermore, good pregnancy rates were achieved from early on in the studied population.

An important factor was that patients tried to achieve pregnancy spontaneously for variable periods of time and tried different numbers of treatment cycles by gonadotropin ovarian stimulation with IUI. However, over a period of 12-month follow-up, 60 patients achieved pregnancies, which represents an overall pregnancy rate of $39 \%$ per patient, which is consistent with prior reports. Higher crude pregnancy rate and CCR can be achieved if COS + IUI is used, as was demonstrated in our study. Similar finding was reported by Tummon et al. in a randomized controlled trial for treatment of infertility associated with early stages of endometriosis, in which he concluded that IUI and controlled ovarian hyperstimulation was associated with superior outcome [12].

\section{Conclusion}

In conclusion, the findings of this study suggest that, in infertile women with endometriosis, both techniques of fimbrioplasty (suturing and flowering) are associated with comparable outcomes. Since the flowering technique is simpler and easier to learn and requires less operative time, it should be the technique of choice in such patients.

Acknowledgments The authors wish to thank Jenny LaChance and Cheryl Anderson for their assistance in the preparation of this manuscript. This work has been presented in the AAGL-35th Annual Meeting, Chicago, IL, USA 2005.

\section{References}

1. Khalaf Y (2003) ABC of subfertility. Tubal subfertility. BMJ 13 (327):610-613

2. Hull MG, Glazener CM, Kelly NJ, Conway DI, Foster PA, Hinton RA et al (1985) Population study of causes, treatment, and outcome of infertility. BMJ 291:1693-1697

3. Gomel V (1983) Microsurgery in female infertility, vol. 1. Little Brown, Boston

4. Audebert AJ, Pouly JL, Von Theobald P (1998) Laparoscopic fimbrioplasty: an evaluation of 35 cases. Hum Reprod 13:1496-1499

5. Audebert A, Cognat M, Cittadini E (1998) Microchirurgie en Infertilite', vol. 1. Cofese, 1980, Palerme, Italy

6. American Society for Reproductive Medicine (1997) Revised American Society for Reproductive Medicine classification of endometriosis: 1996. Fertil Steril 67:817-821

7. Canis M, Mage G, Pouly JL, Manhes H, Wattiez A, Bruhat MA (1991) Laparoscopic distal tuboplasty: report of 87 cases and a 4-year experience. Fertil Steril 56:616-621

8. Dlugi AM, Reddy S, Saleh WA, Mersol-Barg MS, Jacobsen G (1994) Pregnancy rates after operative endoscopic treatment of total (neosalpingostomy) or near total (salpingostomy) distal tubal occlusion. Fertil Steril 62:913-920

9. Gomel V (1977) Salpingostomy by laparoscopy. J Reprod Med $18: 265-269$

10. Patton GW (1982) Pregnancy outcome following microsurgical fimbrioplasty. Fertil Steril 37:150-155

11. Abuzeid MI, Mitwally MF, Abeer AI, Formentini E, Ashraf M, Abuzeid O, Diamond MP (2007) The prevalence of fimbrial pathology in patients with early stages of endometriosis. J Min Inv Gynec 14:49-53

12. Tummon IS, Asher LJ, Martin JSB, Tulandi T (1997) Randomized controlled trial of superovulation and insemination for infertility associated with minimal or mild endometriosis. Fertil Steril 68:8-12 